\title{
ASPECTOS ECOLÓGICOS DE LA ANIDACIÓN DE CROCODYLUS ACUTUS (REPTILIA: CROCODYLIDAE) EN DOS LOCALIDADES DE LA COSTA DE OAXACA, MÉXICO
}

\author{
CÉSAR CEDILLO LEAL ${ }^{1}$, JESÚS GARCÍA GRAJALES ${ }^{2}$, JUAN \\ CARLOSMARTÍNEZ GONZÁLEZ ${ }^{1}$, FLORENCIO BRIONES \\ ENCINIA $^{1}$ \& EUGENIA CIENFUEGOS RIVAS ${ }^{1^{*}}$ \\ ${ }^{1}$ Facultad de Ingeniería y Ciencias, Universidad Autónoma de Tamaulipas, Centro Universitario \\ Adolfo López Mateos, Ciudad Victoria, Tamaulipas, México. Fax: +52 834318 1721; mvz_cedillo@ \\ hotmail.com,jmartinez@uat.edu.mx,fbriones@uat.edu.mx, ecienfue@uat.edu.mx \\ ${ }^{2}$ Universidad del MAR, Campus Puerto Escondido, Instituto de Recursos, km 3.5 carretera Puerto \\ Escondido-Sola de Vega, San Pedro Mixtepec, Oaxaca, México; archosaurio@yahoo.com.mx \\ * Autor de correspondencia: Eugenia Cienfuegos Rivas<ecienfue@uat.edu.mx>
}

Cedillo Leal, C., García Grajales, J., Martínez González, J. C., Briones Encinia, F.\& Cienfuegos

Rivas, E. 2013. Aspectos ecológicos de la anidación de Crocodylus acutus (Reptilia: Crocodylidae) en dos localidades de la costa de Oaxaca, México. Acta Zoológica Mexicana (n. s.), 29(1): 164-177.

RESUMEN. La anidación es uno de los periodos del ciclo reproductivo de los cocodrilos que tiene mayor impacto sobre la sobrevivencia de la especie, por lo que el objetivo del presente estudio fue describir los aspectos ecológicos de la anidación del cocodrilo americano (Crocodylus acutus) en la costa de Oaxaca, México. Se evaluaron nueve nidadas distribuidas en dos localidades (La Ventanilla $\mathrm{n}=6$; Palmasola $\mathrm{n}=3$ ). Los registros de campo incluyeron fechas de ovoposición y de eclosión, mediciones externas (distancia del centro del nido al árbol y a la orilla de agua más cercana, la cobertura dosel), e internas del nido (profundidad del tope del nido, profundidad y ancho de la cámara de incubación, humedad y pH del tope y de la cámara de incubación) así como característicasdelos huevos (número, fertilidad, largo, ancho y peso). Se realizó la prueba de Mann-Whitney a las variables internas y externas del nido y a las variables detamaño y fertilidadde las nidadas entre localidades. Se realizó un modelo lineal anidado a las medidas de ancho, largo y peso delos huevos. Las variables externas del nido no presentaron diferencias significativas entre localidades $(\mathrm{p}>0.05)$.Para las características internas delos nidos se observó una sola diferenciasignificativa $(\mathrm{p}=0.04)$ a nivel del $\mathrm{pH}$ en el tope del nido (6.71vs 6.01), al igual quepara las variables ancho (4.60 vs $4.84 \mathrm{~cm})$, largo $(7.54 \mathrm{vs} 7.66 \mathrm{~cm})$ y peso $(95.4 \mathrm{vs} 104.4 \mathrm{~g}) \mathrm{de}$ los huevos. Este estudio brindalos primeros datos sobre la ecología de anidación del cocodrilo americano en Oaxaca y los resultados obtenidos sugieren un buen estado reproductivo en la región. Sin embargo, es necesaria la información de largo plazo para aumentar el tamaño de la muestra y confirmar el estado reproductivo de la especie.

Palabras claves: Crocodylus acutus, ecología de anidación, Oaxaca

Recibido:11/06/2012; aceptado: 09/01/2013. 
Cedillo Leal, C., García Grajales, J., Martínez González, J. C., Briones Encinia, F. \& Cienfuegos Rivas, E. 2013.Nesting ecology of Crocodylus acutus (Reptilia: Crocodylidae) in two locations of the coast of Oaxaca, Mexico.Acta ZoológicaMexicana (n. s.), 29(1): 164-177.

ABSTRACT.Nesting is a part of the reproductive cycle of crocodiles with the greatest impact on the survival of the species, so the objective of this study was to describe ecological aspects ofthe American crocodile (Crocodylus acutus)nesting off the coast of Oaxaca, Mexico Nine nests from two localities (La Ventanillan=6; Palmasola $n=3$ ) were evaluated. Field records included oviposition and hatching dates, external (distance from the center of the nest to the nearest tree and water's edge, canopy cover) and internal (depth of the top of the nest, depth and width of the eggs chamber, humidity and $\mathrm{pH}$ of the top eggs chamber) nest measurements and eggs characteristics (number, fertility, length, width and weight). A Mann-Whitney test was used to compare internal and external nest variables clutches fertility and clutch size between nesting sites. A nested linear model was used to compare mean values of width, length and weight of the eggs between nesting sites. External nest variables were not significantly different ( $\mathrm{p}$ $>0.05)$. For inside variables, we observed only one significant difference $(p=0.04)$, for themeanpH at the top of the nest (6.71 vs 6.01). For egg's characteristics, means of width (4.60 vs 4.84 ), length (7.54 vs $7.66 \mathrm{~cm}$ ) and weight (95.4 vs $104.4 \mathrm{~g}$ ) of the eggs were significantly different between nesting sites. This study brings first data on nesting ecology of the American crocodile in Oaxaca and results obtained suggest a good reproductive status in the region. However, it is needed several years of data to increment the sample size and confirm the specie reproductive status.

Keywords:C.acutus, nesting ecology, Oaxaca

\section{INTRODUCCIÓN}

El cocodrilo americano (Crocodylus acutus) es una de las tres especies existentes en México, sujetas a protección especial (NOM-059-SEMARNAT-2010, Secretaría de Medio Ambiente y Recursos Naturales 2010) del orden Crocodylia. Esta especie es considerada costera (Thorbjarnarson 1989, Mazzotti 1999), se distribuye desde el sur de los Estados Unidos, hasta Venezuela y en el norte de Perú y en las islas caribeñas incluyendo Cuba, Jamaica y Haití (Ernst et al. 1999, Thorbjarnarson 2010). En México, se encuentra en la costa Pacífica desde Sinaloa hasta el estado de Chiapas y en la costa Atlántica desde la reserva de la Biosfera Río Lagartos en Yucatán hasta la Bahía de Chetumal en Quintana Roo (Sigler 2002).

En general, la sobrevivencia de los cocodrilos es afectada por factores como la infertilidad, las variaciones de temperatura y de humedad ambiental, las inundaciones, la depredación, la contaminación y el disturbio humano, siendo este último el que ha ocasionado la destrucción y pérdida de su hábitat (Thorbjarnarson et al. 2006). Asimismo, estos factores afectan desfavorablemente las zonas de reproducción, sitios de anidación y lugares de crianza de C. acutus (Cedeño-Vázquez et al. 2006) y por consecuencia la dinámica y tamaño poblacional de la especie. Por lo tanto la etapa de anidación es la más vulnerable en su ciclo de vida como lo reportan estudios sobre reproducción y anidación de C. acutus en Florida (Mazzotti 1999), Belice (Platt \& Thorbjarnarson 2000a, 2000b), Cuba (Alonso-Tabet et al. 2000), Haití (Thorbjarnarson 1988), República Dominicana (Schubert \& Méndez 2000); y en México como los 
realizados en el estero Boca Negra (Cupul-Magaña et al. 2002), en el río Cuitzmala (Casas-Andreu 2003), en la Reserva de la Biosfera Chamela-Cuixmala (ValtierraAzotla 2007) en las costas de Jalisco y en la Reserva de la Biósfera Banco Chinchorro, Quintana Roo (Charruau et al. 2010).

Así mismo, las características del sitio de anidación pueden afectar el éxito de eclosión (Valenzuela \& Janzen 2001, Doody et al. 2006), ya que se ha encontrado que diferencias en el tipo de suelo, material vegetal utilizado para su construcción, la distancia al agua, la altura sobre el agua, el $\mathrm{pH}$, la humedad del suelo y la cobertura del dosel, tienen efecto sobre la temperatura de incubación, la proporción sexual, el éxito de la nidada y la supervivencia de las crías (Alfonso-Tabet et al. 2000, CasasAndreu 2003, Charruau et al. 2010, Charruau 2012). Crocodylus acutus puede construir dos tipos de nidos: el nido tipo montículo y el nido tipo hueco, siendo este el más común (Greer 1970, Campbell 1972). Esta especie puede también anidar de manera gregaria o colonial como se observó en Jalisco y Cuba (Alonso-Tabet et al. 2000, Rodríguez-Soberón et al. 2002, Valtierra-Azotla 2007) donde las hembras seleccionan áreas elevadas, protegidas del viento y mareas para evitar los riesgos de inundación (Thorbjarnarson 1988) incrementando así el éxito de la eclosión.

Por lo anterior, el objetivo del presente trabajo fue describir los aspectos ecológicos de la anidación de C. acutus en dos localidades de la costa de Oaxaca, México.

\section{MATERIALES Y MÉTODOS}

Zona de estudio. El estudio se llevó a cabo de enero a julio de 2011 en dos localidades de la costa central de Oaxaca. La primera localidad se ubica a $260 \mathrm{~km}$ de la ciudad de Oaxaca en el estero La Ventanilla (Fig. 1). El estero se alimenta al poniente a través del río Tonameca, al norte con filtraciones subterráneas y al nororiente con

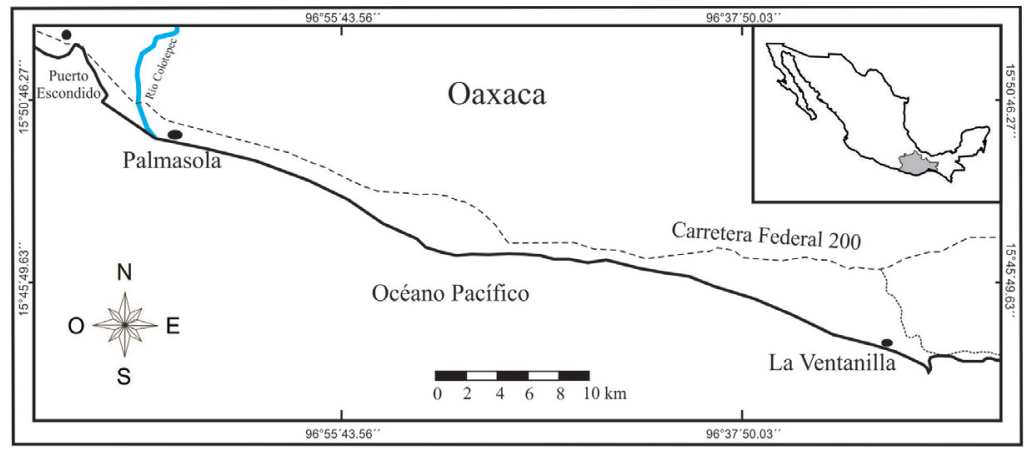

Figura 1. Ubicación geográfica del estero La Ventanilla y la laguna Palmasola en la costa central de Oaxaca, México. 
la cuenca de este río, el cual abarca 57000 ha de superficie. Presenta una boca-barra arenosa que se abre y cierra en los meses de junio-julio y/o diciembre-enero dependiendo de la precipitación y del intervalo de mareas, que es de 1.4 a $1.8 \mathrm{~m}$ (Hubbs \& Roden 1964). La segunda localidad corresponde a la laguna Palmasola ubicada a 12 $\mathrm{km}$ al este de Puerto Escondido, Oaxaca (Fig. 1). La laguna se alimenta del afluente del río Colotepec y mantiene comunicación temporal con el mar a través de la bocabarra en los meses de junio y julio.

Método. Entre enero y febrero de 2011 se realizaron prospecciones en los sitios de estudio para localizar áreas de anidación y determinar algunas características de las mismas. Asimismo, se realizaron recorridos a pie en las áreas localizadas para detectar indicios de actividad de anidación de las hembras, como huellas y rastros de cuerpo entre la orilla del agua y el sitio de anidación. Los nidos se identificaron de dos formas; por medio de los movimientos y excavaciones realizadas por las hembras y a través de la introducción en la tierra de una vara delgada (lo cual implica la ruptura en la mayoría de los casos de uno a dos huevos).

Una vez detectado el nido, con ayuda de un flexometro de $50 \mathrm{~m}$ de longitud se tomaron medidas externas: la distancia del centro del nido a los dos árboles o arbustos más cercanos (DNAr1 y DNAr2); la distancia del centro del nido al cuerpo de agua más cercano (DNAg); así como la cobertura del dosel (CD), marcando una circunferencia de $2 \mathrm{~m}$ de diámetro, partiéndola en cuadrantes y midiendo la sombra proyectada por la vegetación alrededor de las $12 \mathrm{~h}$ del día. Posteriormente, se tomaron medidas internas del nido el cual fue excavado con cuidado y se le dividió en dos partes para su medición: el tope, medido desde la superficie del suelo al primer huevo; y la cámara de incubación, medido desde el primer huevo hasta el fondo del nido. A estas partes se les tomó medidas de profundidad del tope del nido (PTN), y profundidad de la cámara de incubación(PCIN), así como el ancho superior de la cámara de incubación del nido (ACIN1) y el ancho inferior de la misma(ACIN2). Adicionalmente, se colectaron muestras de suelo (500g) de cada nido para analizar el porcentaje de arena, limo y arcilla, estimar el $\mathrm{pH}$ del tope ( $\mathrm{pH}-\mathrm{T}$ ) y de la cámara de incubación ( $\mathrm{pH}-\mathrm{CI}$ ) y calcular la humedad relativa del tope (HUM-T) y de la cámara de incubación (HUM-CI). El análisis de granulometría se realizó utilizando el método de Bouyoucos (1936), el cual consiste en colocar $40 \mathrm{~g}$ de suelo en $100 \mathrm{ml}$ de solución calgon dejándola reposar durante 10 minutos para después mezclarla durante 5 minutos y colocarla en una probeta agregándole $1000 \mathrm{ml}$ de agua destilada, posteriormente se introduce el densímetro y se toman lecturas a los 30 segundos, 1 , 10 y 30 minutos, y a las $2 \mathrm{hrs}$; para posteriormente obtener el tipo de sustrato de la muestra procesada. El pH-T y el pH-CI se estimaron con la técnica sugerida por González-Fernández et al. (2003).Se calcularon HUM-T y HUM-CI colocando las muestras frescas de suelo en una estufa de secado a $110^{\circ} \mathrm{C}$ por $24 \mathrm{~h}$, obteniéndose el valor en porcentaje (\%) por diferencia del peso de la muestra fresca menos el peso de la muestra seca. 
Los huevos fueron marcados cuidadosamente en la superficie dorsal con un lápiz durante el proceso de excavación y medición con la finalidad de asegurar que al regresarlos a la cámara de incubación se mantuviera la orientación y posición original. Para la manipulación de los huevos se utilizaron guantes de latex y charolas plásticas para evitar dejar rastros y olores en los huevos y en los sitiosdonde están ubicados los nidos. Se determinó el número de huevos $(\mathrm{NH})$ en cada nidoy a cada huevo se le tomó el largo $(\mathrm{LH})$ y el ancho $(\mathrm{AH})$ con un vernier electrónico (Digital Caliper ${ }^{\circledR}$, con precisión de $\pm 0.1 \mathrm{~mm}$ ), así como el peso $(\mathrm{PH})$ con una báscula digital (marca Digital ocketScale ${ }^{\circledR}$ de $0.1 \mathrm{~g}$ de precisión). Además, se determinó la viabilidad (fertilidad) de los huevos (FH) mediante la presencia de la banda de desarrollo del embrión (Ferguson 1985).

Siguiendo las definiciones de Rodríguez-Soberón et al. (2002), se consideró como sitiode anidación aislada a aquel sitio en donde se encontró ubicado un nido en particular, como área de anidación gregaria aquel área con características uniformes donde se encontraron dos o más nidos a no más de $100 \mathrm{~m}$ de distancia; nido exitoso aquel en que nació al menos una cría y nido fallidoaquel que no presentó ninguna cría eclosionada.

Al final del periodo de incubación, se realizaron visitas frecuentes a cada sitio y área de anidación para registrar las fechas exactas de laseclosionesde cada nido así como el éxito delmismo.

Análisis estadístico.Se aplicó la prueba no paramétrica de Mann-Whitney (Zar 1999) a las variables internas y externas del nido y a las variables de número de huevos y fertilidad del huevo entre localidades con el programa SAS ${ }^{\circledR}$ 9.1.3.

Se utilizó un modelo lineal general y la prueba de comparación de medias de Tukey $(\alpha=0.05)$ para detectar diferencias entre localidades a nivel de las variables $\mathrm{AH}, \mathrm{LH}$ y $\mathrm{PH}$.

Modelo lineal general:

$$
\mathrm{Y}_{\mathrm{ijk}}=\mu+\mathrm{L}_{\mathrm{i}}+\mathrm{N}(\mathrm{L})_{\mathrm{j}(\mathrm{i})}+\mathrm{E}_{\mathrm{ijk}}
$$

Donde: $\mathrm{Y}_{\mathrm{ijk}}=$ ancho, largo y peso de los huevos

$\mu=$ efecto de la media general

$\mathrm{L}_{\mathrm{i}}=$ efecto de la i-ésima localidad

$\mathrm{N}(\mathrm{L})_{\mathrm{j}(\mathrm{i})}=$ efecto anidado del j-ésimo nido dentro de la i-ésima localidad

$\mathrm{E}_{\mathrm{ijk}}=$ Error aleatorio.

\section{RESULTADOS}

Descripción de la anidación.Se registró un total de nueve nidos, todos ellos tipo hueco, seis en el estero La Ventanilla y tres en la laguna Palmasola. En La Ventanilla 
el periodo de oviposición fue de 16 días (Cuadro 1) y los nidos se distribuyeron en dos áreas de anidación gregaria (2 nidos por área) con seis sitios de anidación.La vegetación predominante fue la palma de coco (Cocos nucifera), la palma real (Roystonea regia) y el mangle rojo (Rhizophora mangle) observándose la presencia de raíces en todos los nidos. En Palmasola el periodo de oviposición fue de 8 días (Cuadro 1), y se detectaron tres sitios de anidación aislados.La vegetación predominante fue el mangle blanco (Laguncularia racemosa) asociado con guamúchil (Cordia alliodora), guayacán (Caesalpinia paraguariensis) y huizache (Acacia pennatula); también se observó la presencia de cantos rodados (piedra bola) en el interior de los nidos.

El periodo de incubación promedio para las dos localidades fue de $77.1 \pm 8.69$ días(n=6, Cuadro 1) Se descartó la información del nido 4 el cual se incubó artificialmente debido a que los habitantes de la comunidad de La Ventanilla decidieron extraerlo por temor a la depredación por mapaches. El periodo de eclosiones para las dos localidades duró 17 días (Cuadro 1). Los sitios de anidación escogidos por las hembras fueron lugares elevados protegidos de los vientos y mareas por la presencia de mangle, palmeras y arbustos. Además, los nidos encontrados en ambas localidades presentaron un porcentaje menor de limo y arcilla y un mayor porcentaje de arena, por lo que se les consideró de sustrato arenoso (Cuadro 2).

En la localidad de Palmasola se observaron dos nidos fallidos debido a que se inundaron por la presencia del fenómeno natural "Mar de fondo" (sistema de olas regular y con crestas suaves con ausencia de vientos, el cual se desplaza fuera de la zona de generación y llega a lugares muy lejanos) del 23 de mayo al 2 de junio del 2011 en las costas oaxaqueñas (Crónicas de Oaxaca 2011). En Palmasola se encontraron cinco nidos intactos de temporadas anteriores (i.e., fallidos). De esos cinco sitios

Cuadro 1. Fechas de oviposición y de eclosión y días de incubación delos nidos de cocodrilo americano en dos localidades de la costa de Oaxaca, México. * Dato no incluido en la estimación del promedio de días de incubación (ver discusión).

\begin{tabular}{ccccc}
\hline Localidad & Nido & $\begin{array}{c}\text { Fecha } \\
\text { de oviposición }\end{array}$ & $\begin{array}{c}\text { Fecha } \\
\text { de eclosión }\end{array}$ & $\begin{array}{c}\text { Días } \\
\text { de incubación }\end{array}$ \\
\hline Laguna Palmasola & 1 & 16-Marzo & - & - \\
& 2 & 16-Marzo & 27-Mayo & 72 \\
Estero La Ventanilla & 3 & 21-Marzo & - & - \\
& $4^{*}$ & 8-Marzo & 11-Junio & $95^{*}$ \\
& 5 & 8-Marzo & 22-Mayo & 75 \\
& 6 & 10-Marzo & 29-Mayo & 80 \\
& 7 & 19-Marzo & 1-Junio & 74 \\
& 8 & 22-Marzo & 7-Junio & 76 \\
& 9 & 29-Marzo & 5-Junio & 68 \\
\hline
\end{tabular}


Cuadro 2. Tipo de sustratos (\%) de los nidos del cocodrilo americano en Palmasola (*) y La Ventanilla $(\boldsymbol{\Delta})$.

\begin{tabular}{|c|c|c|c|c|}
\hline \multirow[b]{2}{*}{ Nido } & & \multicolumn{3}{|c|}{ Textura (\%) } \\
\hline & & Arena & Limo & Limo-Arcilla \\
\hline \multirow[t]{2}{*}{$1 *$} & Superficie del nido & 95.11 & 1.80 & 3.09 \\
\hline & Cámara de Incubación & 94.24 & 2.74 & 3.02 \\
\hline \multirow[t]{2}{*}{$2 *$} & Superficie del nido & 95.32 & 1.80 & 2.88 \\
\hline & Cámara de Incubación & 95.32 & 1.73 & 2.95 \\
\hline \multirow[t]{2}{*}{$5 \Delta$} & Superficie del nido & 96.26 & 0.72 & 3.02 \\
\hline & Cámara de Incubación & 96.33 & 0.79 & 2.88 \\
\hline \multirow[t]{2}{*}{$6 \boldsymbol{\Delta}$} & Superficie del nido & 96.4 & 0.72 & 2.88 \\
\hline & Cámara de Incubación & 96.55 & 0.50 & 2.95 \\
\hline \multirow[t]{2}{*}{$7 \boldsymbol{\Delta}$} & Superficie del nido & 96.26 & 0.86 & 2.88 \\
\hline & Cámara de Incubación & 96.12 & 0.86 & 3.02 \\
\hline \multirow[t]{2}{*}{$8 \boldsymbol{\Delta}$} & Superficie del nido & 96.4 & 0.80 & 2.80 \\
\hline & Cámara de Incubación & 96.19 & 0.93 & 2.88 \\
\hline \multirow[t]{2}{*}{$9 \boldsymbol{\Delta}$} & Superficie del nido & 94.96 & 2.16 & 2.88 \\
\hline & Cámara de Incubación & 94.96 & 2.16 & 2.88 \\
\hline
\end{tabular}

de anidación, cuatro fueron visitados por cocodrilos este año (2011)de los cuales uno presentó oviposición a unos cuantos metros del nido viejo. El nido restante no fue visitado por cocodrilos durante el periodo de estudio.

En La Ventanilla se presentó la depredación de un nido con la pérdida de un huevo solamente y en Palmasola se registró la pérdida de nueve huevos en un nido debido al intento de anidación de una iguana verde (Iguana iguana) en el mismo sitio.

Aspectos ecológicos de la anidación.Las variables DNAr1 (1.45 \pm 0.59m), DNAr2 $(3.74 \pm 2.14 \mathrm{~m})$, DNAg $(11.28 \pm 3.63 \mathrm{~m}), \mathrm{CD}(23.68 \pm 15.04 \%), \mathrm{PTN}(24.53 \pm 8.50$ $\mathrm{cm}), \operatorname{PCIN}(28.76 \pm 6.18 \mathrm{~cm})$, ACIN1 $(33.57 \pm 9.81 \mathrm{~cm})$, ACIN2 $37.28 \pm 9.58 \mathrm{~cm})$, $\mathrm{pH}-\mathrm{T}(6.20 \pm 0.60), \mathrm{pH}-\mathrm{CI}(6.32 \pm 0.44), \mathrm{HUM}-\mathrm{T}(4.79 \pm 3.19 \%)$ y HUM-CI $(5.78 \pm$ $4.17 \%$ ) fueron similares ( $p>0.05$, Cuadro 3 ) entre las localidades de La Ventanilla y Palmasola. La única variable que mostró diferencia significativa entre localidades fue pH-T (6.20 $\pm 0.6 ; \mathrm{p}=0.04$, Cuadro 3).

Características de los huevos. Se contabilizaron un total de 318 huevos entre las dos localidades. En La Ventanilla la producción fue de 215 huevos, de los cuales 195 (90.7\%) fueron fértiles, 16 (7.4\%) infértiles y 4 (1.9\%) sufrieron ruptura durante la búsqueda del nido (véase metodología). El éxito de eclosión fue de 75.60\%. En Pal- 
masola la producción fue de 103 huevos, registrándose $92(89.3 \%)$ huevos fértiles, $9(8.7 \%)$ infértiles y $2(1.9 \%)$ sufrieron ruptura durante la búsqueda delnido (véase metodología); con un éxito de eclosión del $72.20 \%$.

El NH y la FH promedio por nido fueron de $35.3 \pm 9.95$ huevos y $93 \pm 26 \%$, respectivamente. Esas variables no presentaron diferencia significativa $(\mathrm{p}>0.05)$ entre localidades. Las variables AH $(4.76 \pm 0.21 \mathrm{~cm}), \mathrm{LH}(7.62 \pm 0.27 \mathrm{~cm})$ y PH $(101.51 \pm$ $7.91 \mathrm{~g})$ presentaron diferencia significativa $(\mathrm{p}<0.05)$ entre localidades. Los huevos de los nidos de La Ventanilla fueron más grandes y pesados que los huevos de los nidos de Palmasola (Cuadro 4).

Cuadro 3. Media y desviación estándar (DE) de las variables internas y externas de los nidos de cocodrilo americano Palmasola y La Ventanilla, Oaxaca, México. $\mathrm{N}$ = número de nidos por localidad.

\begin{tabular}{|c|c|c|c|c|c|}
\hline & & $\begin{array}{c}\text { Laguna } \\
\text { Palmasola }\end{array}$ & & $\begin{array}{c}\text { Estero } \\
\text { Ventanilla } \\
\end{array}$ & $\begin{array}{c}\text { Prueba } \\
\text { Mann-Whitney }\end{array}$ \\
\hline Variable & $\mathrm{N}$ & Media (DE) & $\mathrm{N}$ & Media (DE) & $\mathrm{p}$ \\
\hline Distancia nido-árbol 1 (m) & 3 & $1.62(0.20)$ & 6 & $1.36(0.70)$ & 0.76 \\
\hline Distancia nido-árbol 2 (m) & 3 & $2.60(1.46)$ & 6 & $4.35(2.19)$ & 0.36 \\
\hline Distancia nido-agua (m) & 3 & $10.84(3.47)$ & 6 & $11.51(3.60$ & 1.00 \\
\hline Cobertura dosel (\%) & 3 & - & 6 & $23.68(15.04)$ & \\
\hline Profundidad tope del nido $(\mathrm{cm})$ & 3 & $24.40(9.19)$ & 6 & $24.60(8.14)$ & 0.88 \\
\hline Profundidad cámara de incubación nido $(\mathrm{cm})$ & 3 & $30.48(7.81)$ & 6 & $27.85(4.89)$ & 0.55 \\
\hline Ancho cámara de incubación 1 (cm) & 3 & $29.82(5.15)$ & 6 & $35.56(11.05)$ & 1.00 \\
\hline Ancho cámara incubación $2(\mathrm{~cm})$ & 3 & $34.23(4.19)$ & 6 & $38.90(11.13)$ & 0.88 \\
\hline $\mathrm{pH}$ del tope del nido & 3 & $6.71(0.08)$ & 6 & $6.01(0.59)$ & 0.04 \\
\hline $\mathrm{pH}$ de la cámara incubación del nido & 3 & $6.46(0.18)$ & 6 & $6.26(0.49)$ & 1.00 \\
\hline Humedad del tope del nido (\%) & 3 & $6.10(4.97)$ & 6 & $4.31(2.01)$ & 0.84 \\
\hline Humedad cámara incubación del nido (\%) & 3 & $9.75(5.84)$ & 6 & $4.30(1.81)$ & 0.56 \\
\hline
\end{tabular}

Cuadro 4. Media y desviación estándar (DE) delas características de las nidadas del cocodrilo americano en Palmasola y La Ventanilla, Oaxaca, México.N = Número de nidos.

\begin{tabular}{|c|c|c|c|c|}
\hline \multirow[b]{2}{*}{ Variables } & \multicolumn{2}{|c|}{ Laguna Palmasola } & \multicolumn{2}{|c|}{ Estero Ventanilla } \\
\hline & $\mathrm{N}$ & Media (DE) & $\mathrm{N}$ & Media (DE) \\
\hline * Número de huevos & 3 & $34.67(3.49)^{\mathrm{a}}$ & 6 & $39.34(10.33)^{\mathrm{a}}$ \\
\hline * Fertilidad del huevo (\%) & 3 & $0.90(0.30)^{\mathrm{a}}$ & 6 & $0.94(0.24)^{\mathrm{a}}$ \\
\hline $\boldsymbol{\Delta}$ Ancho del huevo (cm) & 3 & $4.60(0.19)^{\mathrm{a}}$ & 6 & $4.84(0.17)^{\mathrm{b}}$ \\
\hline A Largo del huevo (cm) & 3 & $7.54(0.29)^{\mathrm{a}}$ & 6 & $7.66(0.26)^{\mathrm{b}}$ \\
\hline$\Delta$ Peso del huevo (g) & 3 & $95.54(4.28)^{\mathrm{a}}$ & 6 & $104.40(7.65)^{\mathrm{b}}$ \\
\hline
\end{tabular}




\section{DISCUSIÓN}

Descripción de la anidación.Thorbjarnarson (1989) concluyó que la temporada reproductiva del cocodrilo americanotiene una tendencia latitudinal, ya que esta comienza primero en latitudes bajas y más tarde en latitudes altas.Además,el mismo autor señala que $C$. acutus sigue el patrón típico de las especies con nidos tipo hueco,oviposicionando en la época de sequía y eclosionando en la época de lluvias, reduciendo así la posibilidad de inundaciones de los nidos yaumentando la disponibilidad de alimento para las crías (Cott 1961, Thorbjarnarson 1989). Esta tendencia latitudinal coincide con lo observado en este estudio donde la anidación se llevó a cabo de febrero a marzo, similar a lo reportado por Alonso \& Rodríguez (1998) en Cuba, pero diferente a lo reportado por Thorbjarnarson (1989) en Colombia y parte de Costa Rica (enero - febrero), por Casas-Andreu (2003) en las costas de Jalisco (abril - mayo), por Kushlan (1988) en Florida (abril - mayo); y por Charruau et al. (2010) en Quintana Roo (mayo - junio). Es importante recalcar que el periodo de incubación en las dos localidades de este estudio se encuentra dentro del rango descrito para la especie (Thorbjarnarson 1989).

Ogden (1978) menciona que los nidos tipo montículo son característicos de especies que habitan en sitios pantanosos, mientras que los nidos tipo hueco son característicos de las especies que habitan en playas o zonas arenosas. Esto coincide con lo observado en este estudio y por Casas-Andreu (2003) en Jalisco donde todos los nidos fueron tipo hueco; no obstante la información obtenida en este estudio difiere a lo observado por Mazzotti (1989) quien reporta mayor número de nidos tipo montículo (79\%) y menor número de nidos tipo hueco (21\%) en el Sur de Florida, debido a que la mayoría de los nidos estuvieron colocados a la orilla margosa de los arroyos. En este estudio se encontraron áreas de anidación gregarias lo cual también se ha descrito en el Sur de Florida (Kushlan \& Mazzotti 1989), Cuba (Rodríguez-Soberón et al. 2002) y Jalisco (Valtierra-Azotla 2007).

Para el cocodrilo americano se ha señalado que existe fidelidad de las hembras por los sitios de anidación (Charruau et al. 2010, Charruau \&Hénaut 2012), así como para el cocodrilo del Orinoco, C. intermedius (Thorbjarnarson \& Hernández 1993) y el cocodrilo del Nilo, C. niloticus (Graham et al. 1976).En este estudio se observó actividad (rastros) de hembras en sitios de anidación del año anterior lo que nos sugiere una fidelidad por parte de las hembras y reutilización de los mismos sitios de anidación al observar que un nido en el presente estudio fue colocado al lado de un nido del año anterior.

La depredación no fue un factor para el fracaso de los nidos en estas localidades de la costa de Oaxaca, esto difiere de lo observado por Ogden (1978) y Kushlan \& Mazzotti (1989) en Florida quienes señalan que el 14\% de las nidadas fueron depredadas, principalmente por mapaches (Procyon lotor). La inundación se ha reportado 
como un factor importante de pérdida de los nidos en varias especies de cocodrilianos (Pooley 1969, Webb et al. 1977, Crawshaw \& Schaller 1979, Mangnusson 1982, Mazzotti et al. 1988, Charruau et al. 2010) como lo ocurrido en la localidad de Palmasola, por efecto del fenómeno "Mar de fondo" que se presentó en las costas oaxaqueñas durante el periodo de incubación. El exceso de humedad en el sustrato de los nidos reduce la capacidad de difusión del oxigeno en la superficie del cascarón, causando malformaciones, asfixia y posteriormente la muerte del embrión en desarrollo(Mazotti et al. 1988, Thorbjarnarson 1989,Ji et al. 2002).

Aspectos ecológicos de la anidación. Los promedios de la distancia del nido al árbol más cercano, la distancia del nido a la orilla del agua, la profundidad del nido así como el ancho de la cámara de incubación se encuentran dentro de los intervalos reportados para la especie (Kushlan \& Mazzotti 1989, Thorbjarnarson 1989, Platt \& Thorbjarnarson 2000b, Cupul-Magaña et al. 2002, Casas-Andreu 2003, Charruau et al. 2010).

La humedad encontrada en los nidos fue mayor a lo reportado por Casas-Andreu (2003). Asimismo el pH de los nidos fue menor para la costa de Oaxaca que en Jalisco según lo reportado por Casas-Andreu (2003) esto se puede deber a la diferencia en el tipo de sustrato de los nidos. En este estudio todos los nidos fueron arenosos similar a lo encontrado por Charruau et al. (2010) en Quintana Roo, pero difiere de lo reportado por Casas-Andreu (2003) quien mencionó tres tipos de sustratos: los arenosos, los areno-limosos y los areno-limo-arcillosos en la costa de Jalisco. En el sur de Florida. Por otra parte,Khuslan \& Mazzotti (1989) registraron cuatro tipos de sustratos: arenosos, margosos, turbosos y rocosos.

Mazzotti (1989) señaló que las nidadas pequeñas están asociadas a hembras primerizas (jóvenes), esto podría ser referido para uno de los nidos ( 22 huevos) encontrado en La Ventanilla. Esto es igual a lo descrito por Ferguson (1985) en caimanes, donde la infertilidad y menos cantidad de huevos por nido fueron para hembras jóvenes y seniles.

Características de los huevos. El tamaño promedio de la nidada (35.3 \pm 9.95 huevos) encontrado en este estudio es similar a lo reportado por Kushlan \& Mazzotti (1989) y Valtierra-Azotla (2007), pero menor a lo reportado por Ogden (1978) quien menciona 44 huevos, y mayor que lo publicado por Platt \& Thorbjarnarson (2000b), Cupul-Magaña et al. (2002), Rodríguez-Soberón et al. (2002), Casas-Andreu (2003) y Charruau et al. $(2010,2011)$ quienes reportan un número promedio de huevos por nido entre 16.2 y 27.9 . El tamaño promedio de las nidadas en las costas oaxaqueñasestá entre los mayores registrados para el cocodrilo americano en México y en todo su rango de distribución. La fertilidad de los huevos fue mayor en las costas oaxaqueñas que lo registrado en Jalisco (Casas-Andreu 2003) y en Quintana Roo 
(Charruau et al. 2010). Los promedio de las características de los huevos (e.g., largo, ancho y peso) fueron mayores para las costas oaxaqueñas que los reportados en Belice (Platt \& Thorbjarnarson 2000b), Jalisco (Casas-Andreu 2003) y Quintana Roo (Charruau et al. 2010). El peso promedio de los huevos fue menor a lo reportado por Cupul-Magaña et al. (2002) en el estero Bocanegra, Jalisco. Además, los promedios de largo, ancho y peso de los huevos fueron diferentes entre La Ventanilla y Palmasola, sugiriendopromedios de tamaño y edad de las hembras diferentes entre las dos localidades.

El éxito de eclosión (75.1\%) observado en la costa de Oaxaca fue similar a lo reportado por Rodríguez-Soberón et al. (2002) de 72.3\% y Charruau et al. (2010) de $73.2 \%$; y superior a lo reportado por Ogden (1978) de 57.5\% y Gaby et al. (1985) de $59 \%$.

Este estudio brinda los primeros datos sobre la ecología de anidación del cocodrilo americano en el estado de Oaxaca. Los resultados sugieren un buen estado reproductivo de esta especie en el estado con disponibilidad de los elementos que requiere el cocodrilo americano para anidar. Sin embargo, nuestra información corresponde a un periodo de tiempo muy corto y pocos nidos fueron encontrados, lo que implica tomar estos resultados con cautela. Un estudio de largo plazo podría precisar los resultados aquí obtenidos y dar una visión más precisa de la situación reproductiva del cocodrilo americano en Oaxaca.

AGRADECIMIENTOS.Se agradece el apoyo logístico y financiamientode la Universidad del Mar (UMAR) y los Servicios Ecoturísticos La Ventanilla S.C. de R.L. de C.V. (Ventanilla) a través del convenio UMAR/CONV-INST/PROMODES/04/2010, otorgados al segundo autor (Jesús García Grajales), así como las facilidades otorgadas por la Sociedad Cooperativa de La Ventanilla y Servicios Ecoturísticos Barra de Navidad (Palmasola). También se agradece al CONACYT por la beca de estudios de posgrado otorgada al primer autor (César N. Cedillo Leal) y al Programa de posgrado en Maestría en Ciencias en Sistemas Agropecuarios y Medio Ambiente de la Facultad de Ingeniería y Ciencias de la Universidad Autónoma de Tamaulipas por el apoyo de movilidad académica y soporte técnico. Las observaciones y valiosos comentarios de dos revisores anónimos ayudaron en gran medida a mejorar la calidad de este trabajo.

\section{LITERATURA CITADA}

Alonso, T.A. \& Rodríguez S., R.1998. Observations on the nesting behavior of Crocodylusacutus. Crocodile SpecialistGroupNewsletter, 17: 11-13.

Alonso-Tabet, M., Rodríguez-Soberón, R., Berovides-Álvarez,V.\&Hernández-Fuentes, C. E.2000. Influencia de la geomorfología del hábitat sobre la nidificación de Crocodylus acutus en el refugio de fauna Monte Cabaniguan, Cuba,pp. 42-58. In: Crocodiles: Proceedings of the $15^{\text {th }}$ Working Meeting of the Crocodile Specialist Group, IUCN The World Conservation Union, Gland, Switzerland and Cambridge UK.

Alonso-Tabet, M., Soberón, R., Berovides,V.\& Hernández, C. E.2000. Influence of changes in morphology of nesting habitat on reproduction of Crocodylusacutusin the Monte Cabaniguan Wildlife 
Refuge, Cuba, pp. 42-58. In: Crocodiles: Proceedings of the 15th Working Meeting of the Crocodile Specialist Group, IUCNThe World Conservation Union, Gland, Switzerland and Cambridge UK.

Bouyoucos, G.L.1936. Direction for making mechanical analysis of soils by the hydrometer method. Soil Science, 42: 3.

Campbell, H.W.1972. Ecological or phylogenetic interpretations of crocodilian nesting habits.Nature,238: 404-405.

Casas-Andreu, G. 2003. Ecología de la anidación de Crocodylus acutus (Reptilia: Crocodylidae) en la desembocadura del río Cuitzmala, Jalisco, México. Acta Zoológica Mexicana (n. s.), 89: 111-128.

Cedeño-Vázquez, J.R., Ross,J.P. \& Calmé, S.2006. Population status and distribution of Crocodylusacutusand C. moreletii in southeastern Quintana Roo, México. Herpetological Natural History, 10: $17-30$.

Charruau, P.2012. Microclimate of American crocodile nests in BancoChinchorro Biosphere Reserve, Mexico: Effect on incubation length, embryos survival and hatchlings sex. Journal of Thermal Biology, 37: 6-14.

Charruau, P. \&Hénaut, Y.2012. Nest attendance and hatchling care in wild American crocodiles (Crocodylusacutus) in Quintana Roo, Mexico. Animal Biology, 62: 29-51.

Charruau, P., Méndez de la Cruz,F.R. \& González Cortés, H.2011.Preliminary results on nesting ecology of Crocodylusacutuson Cozumel Island, Mexico. Crocodile Specialist Group Newsletter, 30: 29-32.

Charruau, P., Thorbjarnarson, J.B. \&Hénaut, Y.2010. Tropical cyclones and reproductive ecology of Crocodylusacutus Cuvier, 1807 (Reptilia: Crocodilia: Crocodylidae) on a Caribbean atoll in Mexico. Journal of Natural History, 44: 741-761.

Cott, H.B.1961. Scientific results of an inquiry into the ecology and economic status of the Nile crocodile (Crocodylusniloticus) in Uganda and northern Rhodesia. Transactions of the Zoological Society of London, 29: 211-356.

Crawshaw, P.C. \& Schaller, G. B.1979. Nesting of Paraguayan caimán (Caiman yacaré) in Brazil. Report $\mathrm{N}^{\circ} 11$ to IBDF. 19pp.

Crónicas de Oaxaca.2011. Efectos del fenómeno Mar de fondo en las costas de Oaxaca. Lunes 23 de mayo de 2011. http://www.cronicaoaxaca.info/informaciongeneral/13761-efectos-del-fenomenomar-de-fondo-en-costas-de-oaxaca.html.

Cupul-Magaña, F.G., Rubio-Delgado, A., Reyes-Juárez, A., \& de-Niz Villaseñor, A.2002. Historia Natural del cocodrilo americano (Crocodylus acutus) en el Estero Boca Negra, Jalisco, México: anidación y crecimiento de neonatos. Ciencia y Mar, 8:31-42.

Doody, J.S., Guarino, E., Georges, A., Corey, B., Murray,G. \&Ewert, M.2006. Nest choice compensates for climate effects on sex rations in a lizard whit environmental sex determination. Evolutionary Ecology, 20: 307-330.

Ernst C.H., Ross,F.D. \&Ross, C. A.1999.Crocodylusacutus(Cuvier) American Crocodile. Catalogue of American Amphibians and Reptiles,700: 1-17.

Ferguson, M.W.J.1985. Reproductive biology and embryology of the Crocodilians,pp: 329-500.In: Gans, C., Billet,F. \&Maderson,P.F.A. (Eds.). Biology of the Reptilia.Development A. Wiley, New York.

Gaby, R., McMahon, M.P., Mazzotti, F.J., Gillies,W.N. \& Ross, J. 1985. Ecology of a population of Crocodylusacutusat a power plant site in Florida. Journal of Herpetology, 19: 189-198.

González-Fernández, P., Ordoñez-Fernández, R., Espejo-Serrano,R. \&Peregrini-Alfonso, F.2003. Cambios en el $\mathrm{pH}$ del perfil de un suelo ácido cultivado y enmendado con diversos materiales para incrementar su fertilidad. Estudios de la Zona no saturada del suelo, 6:373-378. 
Graham, A., Patterson,L. \& Graham, J.1976.Investigation of the Okavango as a primary water resource for Botswana. Technical note No. 34, Aerial photographic techniques for monitoring crocodile populations. F.A.O. Dev. Program. 13 pp.

Greer, A.E.1970. Evolutionary and systematic significance of crocodilian nesting habits.Nature,227: 523-524.

Hubbs, C.L. \& Roden, G. I.1964. Oceanography and marine life along the pacific coast of Middle America, pp. 143-186. In: Wauchope, R. \&West,R. C. (Eds.). Handbook of Midddle American Indians. Vol. I. University of Texas Press, Austin.

Ji, X., Qiu,Q.B. \& Doing, C. H.2002. Influence of incubation temperature on hatching success, energy expenditure for embryonic development, and size and morphology of hatchlings in the oriental garden lizard,Calottes versicolor (Agamidae). The Journal of Experimental Zoology, 292: 649-659.

Kushlan, J.A.1988. Conservation and management of the American Crocodile.Environment Management, 12: 777-790.

Kushlan, J.A. \&Mazzotti, F. J.1989. Population ecology of the American Crocodile. Journal of Herpetology, 23: 7-21.

Magnusson, W.E.1982. Mortality of eggs of the crocodile Crocodylusporosus in Northern Australia. Journal of Herpetology, 16: 121-130.

Mazzotti, F.J.1989. Factors affecting the nesting success of the American crocodile, Crocodylusacutus, in Florida bay.Bulletin of Marine Science, 44: 220-228.

Mazzotti, F.J.1999. The American crocodile in Florida Bay.Estuaries, 22: 552-561.

Mazzotti, F.J., Kushlan,J.A. \& Dunbar-Cooper, A.1988. Desiccation and cryptic nest flooding as probable causes of egg mortality in the American crocodile, Crocodylusacutus, in Everglades National Park, Florida. Florida Scientist, 51:65-72.

Ogden, J.C.1978. Status and nesting biology of the American crocodile, Crocodylusacutus(Reptilia, Crocodylidae) in Florida.Journal Herpetology, 12: 183-196.

Platt, S.G. \&Thorbjarnarson, J.B.2000a.Status and conservation of the American crocodile, Crocodylusacutus, in Belize.Biological Conservation, 96: 13-20.

Platt, S.G. \&Thorbjarnarson, J. B.2000b.Nesting ecology of the American crocodile in the coastal zone of Belize.Copeia, 2000: 869-873.

Pooley, A.C.1969.Preliminary studies on the breeding of the Nile crocodile Crocodylusniloticus in $\mathrm{Zu}-$ luland.Lammergeyer, 3: 22-63.

Rodríguez-Soberón, R., Alonso,M. \&Berovides, V.2002. Nidificación del cocodrilo americano (Crocodylus acutus Cuvier) en el refugio de fauna "Monte Cabaniguan", Cuba, pp. 135-156. In: Verdade, L. \&LarrieraPiracicab,A. (Eds.). La conservación y el manejo de caimanes y cocodrilos de América Latina, V.2. C.N. Editorial. São Paulo, Brasil.

Schubert, A. \& Méndez, H.2000. Métodos para estimar el tamaño de la población del Cocodrilo Americano (Crocodylus acutus) en el Lago Enriquillo, República Dominicana,pp. 372-381. In:Crocodiles: Proceedings of the 15th Working Meeting of the Crocodile Specialist Group, IUCN-The World Conservation Union, Gland, Switzerland and Cambridge UK.

SEMARNAT.2010. Norma Oficial Mexicana NOM-059-SEMARNAT-2010, Protección ambientalEspecies nativas de México de flora y fauna silvestres-Categorías de riesgo y especificaciones para su inclusión, exclusión o cambio-Lista de especies. Diario Oficial de la Federación, 6 de marzo de 2002.

Sigler, L.2002. Conservación y manejo de Crocodylusacutus en México,pp.167-184. In: L.M. Verdad e\&LarrieraPiracicab ,A. (Eds.). La conservación y el manejo de caimanes y cocodrilos de América Latina. CN Editorial. Piracicaba, São Paulo, Brasil. 
Thorbjarnarson, J.B. 1988. The status and ecology of the American crocodile in Haiti. Bulletin of the Florida State Museum, 33: 1-86.

Thorbjarnarson, J.B.1989. Ecology of the American Crocodile, Crocodylusacutus, pp. 228-259.In: Crocodiles their ecology, management and conservation. IUCN.Publication New Series.

Thorbjarnarson, J.B.2010. American Crocodile Crocodylusacutus, pp. 46-53.In: S. C. Manolis\& Stevenson,C. (Eds.). Crocodiles. Status Survey and Conservation Action Plan. Third Edition, IUCN Crocodile Specialist Group: Darwin.

Thorbjarnarson, J. \& Hernández, G.1993. Reproductive ecology of the Orinoco Crocodile (Crocodylusintermedius) in Venezuela. Nesting ecology and egg and clutch relationship.Journal of Herpetology,27: 363-370.

Thorbjarnarson, J.B., Mazzotti, F., Sanderson, E., Buitrago, F., Lazcano, M., Minkowski, K., Muñiz, M., Ponce, P., Sigler, L., Soberón, R., Trelancia,A.M. \& Velasco, A. 2006.Regional habitat conservation priorities for the American crocodile. Biological Conservation, 128: 25-36.

Valenzuela, N. \&Janzen, F. J.2001.Nest-Site Philopatry and the Evolution of Temperature-Dependent Sex Determination. EvolutionaryEcologyResearch, 2001: 779-794.

Valtierra-Azotla, A.M.2007. Ecología poblacional y reproductiva del cocodrilo americano (Crocodylus acutus), en la reserve de la biosfera Chamela-Cuixmala. Tesis Maestría. Universidad Michoacana de San Nicolás de Hidalgo. Morelia, Michoacán, México.

Webb,G.J.W., Messel,H. \& Magnusson, W.1977. The nesting of Crocodylusporosusin Arnhem Land, northern Australia. Copeia, 1977: 238-249.

Zar, J.H.1999. Biostatistical analysis. Prentice-Hall Inc. New Jersey. 663 pp. 